\title{
Judicious Antibiotic Use in Community Acquired Staphylococcal Infections
}

\author{
Arti Kapil $^{1}$
}

Received: 3 December 2020 / Accepted: 3 December 2020 / Published online: 7 January 2021

(C) Dr. K C Chaudhuri Foundation 2021

In the article published in this issue "Community-Acquired Case-Control Study" by Ganesan et al. from Department of Pediatrics, PGIMER, Chandigarh, India [1], the authors report a retrospective case control study in acutely ill children carried out at a tertiary care hospital, with an objective of identifying possible clinical and laboratory indicators to discriminate between community-acquired methicillinresistant Staphylococcus aureus (CA-MRSA) vs methicillinsensitive Staphylococcus aureus (MSSA) infections in children presenting to the hospital with staphylococcal infections acquired in the community. The aim is to be able to guide the judicious empirical use of antibiotics, specifically vancomycin, before culture results are available. The study also compared the outcomes in the two groups.

They did not find any statistically significant clinical or laboratory variables that could differentiate CA-MRSA and MSSA infections or outcomes in the two groups. This might be due to retrospective case control study design and the limited number of patients in the two groups. However, such studies need to be undertaken because Staphylococcus aureus (S. aureus) infections especially SSTI followed by invasive infections are increasing in the community [2].

In the study, it is interesting to note that the difference in numbers of MRSA and MSSA that the authors have reported during the $3 \mathrm{y}$ of study period is not statistically significant (they have included all culture-positives out of 131 patients suspected of staphylococcal infections from the community). This could reflect the presence of both in the community to the same extent. If this is the status, then there is not much choice left in deciding the empirical antibiotic in acutely ill children presenting with staphylococcal infections from community but to administer vancomycin, followed by de-escalation, if

Arti Kapil

akapilmicro@aiims.edu

1 Department of Microbiology, All India Institute of Medical Sciences, New Delhi 110029, India needed after culture results, are available. Some additional data could have added value in understanding the extent of the problem - like number of HA-MRSA, total number of patients culture-positive for $S$. aurues, distribution of MRSA over the years etc. There were only 35 patients with MRSA but their subsequent results do not reflect whether they received appropriate antibiotic empirically or subsequent modification was done. Also as suggested by the authors, recommendation for vancomycin and cephelosporins together as empirical choice may not be required as only vancomycin can be used empirically, if prevalence of MRSA in community is high.

Early appropriate antibiotic should be administered as not only we must save vancomycin and linezolid for MRSA infections but also need to remember that $\beta$-lactam antibiotics have a superior activity against S.aureus compared to vancomycin and should be chosen in MSSA infections [3]. Since we do not have adequate data especially from the community on the prevalence of infections caused by CA-MRSA or MSSA, some clinical variables could help in guiding the choice of appropriate antibiotic till culture report is available. There is however the limitation of a retrospective analysis and a clear answer can be obtained from a prospective study. Also vancomycin overuse has now been shown to be responsible for emergence of heterogeneous vancomycin-intermediate S. aureus (hVISA) which could subsequently lead to VISA [4]. Fortunately in India, we do not yet have vancomycin resistance reported in $S$. aureus though creeping minimum inhibitory concentrations (MICs) have been reported. But this needs a close monitoring as clinical failures have been reported.

The CA-MRSA as a distinct entity from HA-MRSA was reported in 1990s. CA-MRSA are frequently susceptible of non-beta-lactam antibiotics, including clindamycin, tetracycline trimethoprim-sulfamethoxazole, often carrying PantonValentine leukocidin (PVL) encoding gene that increases its virulence, while HA-MRSA are more resistant to even nonbeta-lactam antibiotics. This division, however, is becoming 
blurred now as CA-MRSA is spreading in hospitals and developing multidrug resistance due to selective pressure in healthcare settings and HA-MRSA acquiring PVL gene causing more severe infections [5].

Control of infections due to CA-MRSA is a major challenge in the community even as reservoirs of CA-MRSA in animals are also reported. It is difficult to monitor and implement control measures in a community and molecular typing methods are helpful in understanding the spread of these phenotypes and plan-control strategies.

We know that hand hygiene remains as one most important evidence based intervention to control the transmission of S. aureus infections and with COVID-19 protocols, we may find a reduced transmission even for MRSA. Time will only tell!

\section{Compliance with Ethical Standards}

Conflict of Interest None.

\section{References}

1. Ganesan SL, Mehta A, Lakshmikantha K, Jayashree M, Gautam V, Ray P. Community-acquired methicillin-resistant staphylococcus aureus infections in acutely ill children: a retrospective case-control study. Indian J Pediatr. 2020. https://doi.org/10.1007/s12098-02003391-z.

2. Bassetti M, Peghin M, Trecarichi EM, et al. Characteristics of Staphylococcus aureus bacteraemia and predictors of early and late mortality. PIoS one. 2017;12(2):e0170236.

3. Wong D, Wong T, Romney M, Leung V. Comparative effectiveness of $\beta$-lactam versus vancomycin empiric therapy in patients with methicillin-susceptible Staphylococcus aureus (MSSA) bacteremia. Ann Clin Microbiol Antimicrob. 2016;15(1):27.

4. Zhang S, Sun X, Chang W, Dai Y, Ma X. Systematic review and meta-analysis of the epidemiology of vancomycin-intermediate and heterogeneous vancomycin-intermediate Staphylococcus aureus isolates. PLoS One. 2015;10(8):e0136082.

5. Dhawan B, Rao C, Udo EE, Gadepalli R, Vishnubhatla S, Kapil A. Dissemination of methicillin-resistant Staphylococcus aureus SCCmec type IV and SCCmec type V epidemic clones in a tertiary hospital: challenge to infection control. Epidemiol Infect. 2015;143: 343-53.

Publisher's Note Springer Nature remains neutral with regard to jurisdictional claims in published maps and institutional affiliations. 